\title{
Hepatosplenic schistosomiasis: a great neglected disease of the liver
}

\author{
K. S. WARREN 1 \\ From The Rockefeller Foundation, New York
}

SUMMARY Schistosomiasis mansoni and japonica, helminth infections involving the livers of 100 million people, are among the 'great neglected diseases of mankind' on the basis both of their prevalence and the relative lack of interest shown in them by clinicians and biomedical investigators. Schistosomiasis is not only an interesting disease in itself but has the added advantage of providing unique human and animal models of hepatic pathophysiology, immunopathogenesis, and collagen metabolism.

The Rockefeller Foundation has recently embarked upon a new programme devoted to attracting outstanding biomedical research groups into investigating the 'great neglected diseases of mankind'. These diseases are great in terms of the enormous numbers of people suffering from them, and are neglected both financially and scientifically. One major example is schistosomiasis, a liver disease of approximately 100 million people in which annual worldwide research expenditures are probably less than five million dollars and which is virtually ignored by the liver establishment. Almost all individuals infected with Schistosoma mansoni in Africa, South America, and the Caribbean, and with $S$. japonicum in Asia have some degree of liver involvement (Warren, 1973a). Depending on the intensity of infection (worm burdens), up to $30 \%$ of infected populations suffer from significant hepatomegaly; the most advanced form of this disease, hepatosplenomegaly with portal hypertension and oesophageal varices, is probably present in several million people (Kloetzel, 1963; Ongom and Bradley, 1972; Arap Siongok et al., 1976; Lehman et al., 1976).

Monetary neglect is associated with a lack of interest in the major scientific community of clinicians, clinical investigators, and basic scientists specialising in liver diseases. The fifth edition (1975) of Sherlock's great Diseases of the Liver and Biliary System accords schistosomiasis three pages. While

${ }^{1}$ Address for reprint requests: K. S. W., Director, Division of Health Sciences, The Rockefeller Foundation, 1133 Avenue of the Americas New York, New York 10036, USA. the third edition of Schiff's multi-author work Diseases of the Liver (1969) contained less than 150 words on the subject, the fourth edition (1975) did devote about three pages to schistosomiasis $(0.2 \%$ of the text). The brevity of these descriptions is not due to a dearth of literature, as approximately 16000 papers have now been written on the subject (Warren and Newill, 1967; Warren and Hoffman, 1976). National and international meetings of hepatologists rarely contain papers on schistosomiasis and this is related both to a lack of interest by the audience and a relative lack of interesting well-done studies by potential participants. Again, this is probably not due to a lack of patients in some of the liver centres of the developed worldfor example, the large Puerto Rican population of New York City. Nevertheless, much of clinical and scientific value has been published and it appears that activity in the field is accelerating. This is being facilitated by the direct support of the Edna McConnell Clark Foundation for research on schistosomiasis, the development of the tropical diseases research programme of the World Health Organization, and the great neglected diseases programme of the Rockefeller Foundation, and a renaissance of interest in the tropical diseases by the United States government.

While schistosomiasis in and of itself should command the attention of the liver establishment on a reasonable level with alcoholic cirrhosis, liver toxins, hepatitis, biliary disease, etc., there are compelling reasons beyond this in terms of the unique models it provides of several different aspects of liver disease. Physiologically, many patients with advanced schistosomiasis have marked circulatory changes in the absence of significant 
alterations in liver function. As most of the other forms of chronic liver disease include both portalsystemic collateral circulation and liver parenchymal damage, schistosomiasis offers an opportunity to dissect out the effects of each of these factors. Clinical immunology has attracted the interest of many hepatologists. Hepatosplenic schistosomiasis has now been claimed as an immunological disease, and it appears that more is known of its immunopathogenesis than that of any other form of liver disease (Warren, 1975). Finally, pathologically, schistosomiasis is a major cause of liver fibrosis, and biochemists are now using it as a model to study collagen metabolism. On all of these levels, pathophysiologically, immunologically, and biochemically, it should be realised that, in contradistinction to many other forms of liver disease, there are excellent laboratory models of hepatic schistosomiasis in both rodents and primates, with that most versatile of laboratory animals, the mouse, providing models of all three areas mentioned above.

Before going on to describe the state of our knowledge of clinical hepatic schistosomiasis and its pathophysiology, immunopathology, and biochemistry, it is necessary to set the stage by a brief description of the life cycle of schistosomiasis, particularly in relation to the host parasite relationship. Schistosomes are digenetic trematodes, alternating generations between asexual multiplication in snail intermediate hosts and sexual reproduction in mammalian definitive hosts. Cercariae emitted by snails penetrate human skin and the young schistosomes migrate to the lungs and then to the liver where male and female worms mate and move down into the mesenteric venules within which the females produce between 300 and 3000 eggs daily over a period of many years. The eggs secrete enzymes which enable them to pass out of the blood vessels, through the tissues and into the lumen of the gut from whence they are excreted to carry on the life cycle in snails. More than $50 \%$ of the eggs never escape from the body, however, a large proportion of them breaking free in the circulation to be sieved out in the hepatic portal system. It is important to realise that the adult worms do not multiply within mammalian hosts, that patients with schistosomiasis may have worm burdens varying from one to thousands, and that there is an overdispersed distribution of the organisms, with most humans having light infections (Warren, 1973b). Recent field and necropsy studies have shown that hepatosplenic disease tends to cluster in the relatively small proportion of individuals with large numbers of worms (Kloetzel, 1963; Cheever, 1968; Ongom and Bradley, 1972; Arap Siongok et al., 1976; Lehman et al., 1976; Cheever et al., 1977).
Clinical hepatosplenic schistosomiasis and its pathophysiology

The pathology of hepatic schistosomiasis was described in Egypt by Symmers (1904), the great British pathologist, before either its life cycle or clinical aspects were understood. Over a period of many years it became apparent that the syndrome of Egyptian splenomegaly was a form of congestive splenomegaly due to schistosomal fibrosis of the liver. It then became clear that hepatosplenic schistosomiasis with portal hypertension and oesophageal varices was due to a presinusoidal block to portal blood flow. The aetiology of this syndrome was probably as Symmers first suggested, schistosome eggs trapped in the portal venules and the granulomatous inflammation and subsequent fibrosis which developed around them (Warren, 1973a). Under these conditions it was surprising that total liver blood flow remained within normal limits (Coutinho, 1968), but plastic casts of the hepatic vasculature at necropsy have revealed that blockage to portal blood flow was compensated for by arterialisation of the liver (Andrade and Cheever, 1971), maintaining blood flow and parenchymal cell perfusion. Schistosomiasis is quite unique among chronic forms of liver disease, therefore, in that the primary insult to the liver is not to the parenchymal cell followed subsequently by regeneration, fibrosis, and portal hypertension. Thus, in pure hepatosplenic schistosomiasis marked portal hypertension occurs in the absence of gross changes in liver function tests (Warren et al., 1965).

Careful studies have shown that after a severe haematemesis patients with compensated hepatosplenic schistosomiasis have a low mortality and do not go into hepatic coma (Warren and Rebouças, 1964; Sulit et al., 1964); in contrast with those with cirrhosis of the liver, blood ammonia levels after a haematemesis are either normal or only slightly raised (Warren and Rebouças, 1964). Contrary to previous belief, therefore, it was shown that marked portal collateral circulation alone would not result in a systemic rise in toxic substances absorbed from the gastrointestinal tract. Further studies were performed in patients with this syndrome who had suffered a haematemesis and were treated by a portacaval shunt (Warren et al., 1965). Because these patients had excellent liver function before surgery they were considered to be in the good risk group. After surgery, however, their liver function tests became abnormal, as did their ammonia tolerance, and a large proportion of these individuals developed chronic portal systemic encephalopathy (Warren et al., 1965).

Many of the above studies in humans have been 
corroborated in experimental animals. Some chimpanzees with Schistosomiasis mansoni develop typical schistosomal fibrosis and occasional oesophageal varices have been reported, although portal hypertension does not occur in these primates (Sadun et al., 1970). Severe liver fibrosis is much more common in chimpanzees with Schistosomiasis japonica, but again there is no portal hypertension (Von Lichtenberg et al., 1971). Mice with S. mansoni or japonica develop hepatomegaly, splenomegaly, portal hypertension, and oesophageal varices with liver function tests that are relatively unimpaired (Warren and DeWitt, 1958; Warren and Moore, 1966). Total liver blood flow remains within normal limits and microcirculation studies have revealed neovascular formation which is purely arterial in the areas of fibrosis (Bloch et al., 1972).

A major clinical area worthy of more study is the relationship of involvement of the liver in the 100 million cases of schistosomiasis to other hepatic insults to that organ such as hepatitis, malnutrition, liver toxins, and carcinogens. In one of the rare human studies in this area the prevalence of hepatitis B surface antigen was examined in a series of cases of hepatosplenic schistosomiasis as compared with control cases and blood donors in Brazil (Lyra et al., 1976). The patients with hepatosplenic schistosomiasis proved to be persistent carriers for hepatitis B surface antigen in a significantly higher proportion than the other two groups. The cases of hepatosplenic schistosomiasis who were carriers of the antigen had more clinical signs of chronic liver disease and strikingly more chronic inflammation of the portal spaces on liver biopsy. Further work on these problems has been done in experimental animals; hepatitis in mice $\left(\mathrm{MHV}_{3}\right)$ is exacerbated as shown by increased mortality, liver parenchymal cell destruction, and liver virus titres (Warren et al., 1969); malnutrition has a protective effect in that both egg output by the worms and granuloma formation are diminished (Knauft and Warren, 1969; Akpom and Warren, 1975); and schistosomiasis plus a carcinogen results in a greatly increased incidence of hepatomas (Domingo et al., 1967).

\section{Immunopathology}

As mentioned above, hepatosplenic schistosomiasis is due to inflammation and fibrosis in the presinusoidal portal areas of the liver. Investigations in an experimental murine model have unequivocally shown that the parasite factor responsible for these changes is the egg (Warren, 1972). While microcirculation studies revealed that the eggs alone do not significantly obstruct liver blood flow, the large avascular granulomas which develop around them and the subsequent fibrosis are responsible for the pathophysiological consequences of this infection (Bloch et al., 1972). The aetiology of the $S$. mansoni egg granuloma has been studied using a method in which eggs isolated from the livers of infected mice are injected intravenously through a tail vein and spread throughout the pulmonary microvasculature. Measurements of mean granuloma size at various time periods after egg injection have demonstrated anamnestic secondary responses to the eggs, a high degree of specificity of this reaction and its transferability by lymph node or spleen cells but not by serum (Warren et al., 1967). This strong suggestion that the inflammatory reaction $S$. mansoni eggs was an immunological reaction of the delayed, cell-mediated type was confirmed by a continuing series of experiments on immunosuppression of the lesion (Warren, 1978). Thus inhibitors of cell-mediated immunological reactivity such as antilymphocyte serum, neonatal thymectomy (in mice and chickens), Hodgkins-like disease (SJL/L mice), nude mice, and niridazole strongly suppressed granuloma formation, while suppressants of humoral antibody-mediated responses such as chronic $x$-irradiation, bursectomy (in chickens), Friend virus leukemia, antineutrophil serum, cobra venom factor, and anti-mu serum had no effect (Warren, 1978).

The isolation of highly antigenic soluble substances from $S$. mansoni eggs-soluble egg antigens (SEA)facilitated studies of intradermal responses, antibody formation, and in vitro cell-based systems such as lymphocyte blastogenesis and lymphokine production (Boros and Warren, 1970). Correlations were demonstrated among granuloma formation, delayed skin reactions, and in vitro correlates of delayed hypersensitivity (Boros et al., 1973a). Furthermore, granulomas maintained in vitro produced the lymphokines macrophage migration inhibitory factor (MIF) (Boros et al., 1973b) and eosinophil stimulation promoter (ESP) (James and Colley, 1975) but no immunoglobulins (Pelley et al., submitted for publication). The isolation and purification of three antigens from $S$. mansoni eggs has been accomplished (Pelley et al., 1976), and one of these-major serological antigen one $\left(\mathrm{MSA}_{1}\right)_{\text {- }}$-has been shown by inhibition radioimmunoassay to be as stage and species specific as the egg granuloma itself (Hamburger et al., 1976). Recent studies strongly suggest that $\mathrm{MSA}_{1}$, a glycoprotein of molecular weight 50000 , is a major antigen involved in granulomatous hypersensitivity (Pelley and Pelley, 1976).

In 1963 a phenomenon was observed in chronically infected mice in which granulomas around eggs newly arrived in the liver were much smaller than 
the massive inflammatory lesions seen in the earlier stages of infection (Andrade and Warren, 1964). This phenomenon, initially called 'endogenous desensitization' (Domingo and Warren, 1968) and, presently, 'modulation', has been studied in great detail, and two possible mechanısms have been discovered (Warren, 1977). First, suppression of the cell-mediated granulomatous hypersensitivity occurs coincidentally with an exponential rise in antibody levels against both crude SEA and the purified MSA . $_{\text {. }}$ Second, as the granulomas get smaller and smaller the responsiveness of spleen cells to SEA and T cell mitogens progressively changes and finally disappears altogether. Third, the degree of inflammation around the eggs has been suppressed by transfer of either cells or serum (Warren, 1977).

\section{Fibrosis and collagen metabolism}

The marked periportal fibrosis seen in chronic hepatosplenic schistosomiasis was first described by Symmers in 1904. Essentially, little more was learned about fibrosis in schistosomiasis in man until recent clinical investigations in Egypt (Dunn et al., submitted for publication). These were based on previous studies in experimental animals. In murine schistosomiasis the biochemical assessment of liver fibrosis by measuring hydroxyproline levels was first performed more than a decade ago (Cameron and Ganguly, 1964). The amount of collagen in the liver was shown to rise steeply with the onset of egg production and granuloma formation, reaching a steady state from 16 weeks of infection through one year (Warren, 1966). Earlier experiments based on morphological criteria, such as the Mallory trichrome stain for collagen, revealed that when mice were treated in the acute stages of the infection the large fibrotic granulomas eventually disappeared almost completely (Warren, 1962). In subsequent experiments mice with chronic infections were treated and collagen was measured quantitatively; there was no significant decrease in hydroxyproline levels after treatment, and, morphologically, the large acellular bands of fibrous tissue showed little regression (Warren and Klein, 1969). Recently, a major liver research unit which has had a long interest in hepatic fibrosis has adopted murine $S$. mansoni as a model. Collagen synthesis as measured by the formation of radio-labelled proteinbound hydroxyproline was studied in early infections of mice, revealing a high rate of synthesis; a proline analogue, $L$ azetidine 2 carboxylic acid, inhibited this reaction (Dunn et al., 1977). Examination of the biochemical pathways and of the substrates involved have revealed that arginine and not glutamate is the major precursor of the increased free proline pool in the livers of mice with schistosomiasis (Dunn et al., submitted for publication). The role of collagenase in the development of liver fibrosis and the effect of eosinophils on collagen content and synthesis in the granulomas is now being explored. Investigation performed with colleagues in Egypt using wedge liver biopsies from patients with advanced hepatosplenic disease have demonstrated greatly increased collagen content and marked collagen synthesis averaging seven times that of controls (Dunn et al., submitted for publication).

\section{Conclusion}

The prevalence and pathogenicity of $S$. mansoni and japonica should be sufficient reason for hepatologists to work on these major liver diseases. In addition, the excellent, and in some cases unique, models of liver disease afforded by both patients and experimental animals should provide a further incentive to clinical and laboratory investigators. As noted above, the quantity of work on schistosomiasis has been more than adequate. Because eminent scientists of both the developed and developing world have neglected this widespread major disease the quality of work on this problem has suffered. It is hoped that with the new initiatives in funding for research on the great neglected diseases of mankind by the Rockefeller Foundation, the Edna McConnell Clark Foundation, the Wellcome Trust, the World Health Organization, and a variety of national governmental institutions, our knowledge of this major disease of the liver will be greatly enhanced.

\section{References}

Akpom, C. A., and Warren, K. S. (1975). Calorie and protein malnutrition in chronic murine schistosomiasis mansoni: Effect on the parasite and the host. Journal of Infectious Diseases, 132, 6-14.

Andrade, Z. A., and Cheever, A. W. (1971). Alterations of the intrahepatic vasculature in hepatosplenic schistosomiasis mansoni. American Journal of Tropical Medicine and Hygiene, 20, 425-432.

Andrade, Z. A., and Warren, K. S. (1964). Mild prolonged schistosomiasis in mice: alterations in host response with time and the development of portal fibrosis. Transactions of the Royal Society of Tropical Medicine and Hygiene, 58, 53-57.

Arap Siongok, T. K., Mahmoud, A. A. F., Ouma, J. H., Warren, K. S., Muller, A. S., Handa, A. K., and Houser, H. B. (1976). Morbidity in schistosomiasis mansoni in relation to intensity of infection: study of a community in Machakos, Kenya. American Journal of Tropical Medicine and Hygiene, 25, 273-284.

Bloch, E. H., Abdel Wahab, M. F., and Warren, K. S. (1972). In vivo microscopic observations of the pathogenesis and pathophysiology of hepatosplenic schistosomiasis in the mouse liver. American Journal of Tropical Medicine and Hygiene, 21, 546-557.

Boros, D. L., and Warren, K. S. (1970). Delayed hyper- 
sensitivity-type granuloma formation and dermal reaction induced and elicited by a soluble factor isolated from Schistosoma mansoni eggs. Journal of Experimental Medicine, 132, 488-507.

Boros, D. L., Schwartz, H. J., Powell. A. E., and Warren, K. S. (1973a). Delayed hypersensitivity, as manifested by granuloma formation, dermal reactivity, macrophage migration inhibition and lymphocyte transformation, induced and elicited in guinea pigs with soluble antigens of Schistosoma mansoni eggs. Journal of Immunology, 110, 1118-1125.

Boros, D. L., Warren, K. S., and Pelley, R. P. (1973b). The secretion of migration inhibitory factor by intact schistosome egg granulomas maintained in vitro. Nature, 246, 224-226.

Cameron, G. R., and Ganguly, N. C. (1964). An experimental study of the pathogenesis and reversibility of schistosomal hepatic fibrosis. Journal of Pathology and Bacteriology, 87, 217-237.

Cheever, A. W. (1968). A quantitative post-mortem study of schistosomiasis mansoni in man. American Journal of Tropical Medicine and Hygiene, 17, 38-64.

Cheever, A. W., Kamel, I. A., Elwi, A. M., Mosimann, J. E., and Danner, R. (1977). Schistosoma mansoni and $S$. haematobium infections in Egypt. II. Quantitative parasitological findings at necropsy. American Journal of Tropical Medicine and Hygiene, 26, 702-716.

Coutinho, A. (1968). Hemodynamic studies of portal hypertension in schistosomiasis. American Journal of Medicine, 44, 547-556.

Domingo, E. O., and Warren, K. S. (1968). Endogenous desensitization: changing host granulomatous response to schistosome eggs at different stages of infection with Schistosoma mansoni. American Journal of Pathology, 52, 369-377.

Domingo, E. O., Warren, K. S., and Stenger, R. J. (1967). Increased incidence of hepatoma in mice with chronic schistosomiasis mansoni treated with a carcinogen. American Journal of Pathology, 51, 307-321.

Dunn, M. A., Kamel, R., Kamel, I. A., Biempica, L., El Kholy, A., Hait, P. K., Rojkind, M., Warren, K. S., and Mahmoud, A. A. F. Liver collagen synthesis in schistosomiasis. Submitted for publication.

Dunn, M. A., Rojkind, M., Hait, P. K., and Warren, K. S. (1978). Conversion of arginine to proline in murine schistosomiasis. Submitted for publication,

Dunn, M. A., Rojkind, M., Warren, K. S., Hait, P. K., Rifas, L., and Seifter, S. (1977). Liver collagen synthesis in murine schistosomiasis. Journal of Clinical Investigation, 59, 666-674.

Hamburger, J., Pelley, R. P., and Warren, K. S. (1976). Schistosoma mansoni soluble egg antigens: determination of the stage and species specificity of their serological reactivity by radioimmunoassay. Journal of Immunology, 117, 1561-1566.

James, S. L., and Colley, D. G. (1975). Eosinophils and immune mechanisms: production of the lymphokine eosinophil stimulation promoter (ESP) in vitro by isolated intact granulomas. Journal of the Reticuloendothelial Society, 18, 283-293.

Kloetzel, K. (1963). Some quantitative aspects of diagnosis and epidemiology in schistosomiasis mansoni. American Journal of Tropical Medicine and Hygiene, 12, 334-337.

Knauft, R. F., and Warren, K. S. (1969). The effect of calurie and protein malnutrition on both the parasite and the host in acute murine schistosomiasis mansoni. Journal of Infectious Diseases, 120, 560-575.

Lehman, J. S., Jr, Mott, K. E., Morrow, R. H., Jr, Muniz, T. M., and Boyer, M. H. (1976). The intensity and effects of infection with Schistosoma mansoni, in a rural commu- nity in northeast Brazil. American Journal of Tropical Medicine and Hygiene, 25, 285-294.

Lyra, L. G., Rebouças, G., and Andrade, Z. A. (1976). Hepatitis B surface antigen carrier state in hepatosplenic schistosomiasis. Gastroenterology, 71, 641-645.

Ongom, V. L., and Bradley, D. J. (1972). The epidemiology and consequences of Schistosoma mansoni infection in West Nile, Uganda. I. Field studies of a community at Panyagoro. Transactions of the Royal Society of Tropical Medicine and Hygiene, 66, 835-851.

Pelley, R. P., Boros, D. L., and Warren, K. S. (submitted). Lymphokine production and protein synthesis by isolated, cultured Schistosoma mansoni granulomas.

Pelley, R. P., and Pelley, R. J. (1976). S. mansoni soluble egg antigens. IV. Biochemistry and immunochemistry of major serological antigens with particular emphasis on MSA; In Biochemistry of Parasites and Host-Parasite Relationships, pp. 283-290. Edited by H. Van den Bossche. Elsevier/North Holland Biomedical Press: Amsterdam.

Pelley, R. P., Pelley, R. J., Hamburger, J., Peters, P. A., and Warren, K. S. (1976). Schistosoma mansoni soluble egg antigens: I. Identification and purification of three major antigens, and the employment of radioimmunoassay for their further characterization. Journal of Immunology, 117, 1553-1560.

Sadun, E. H., Von Lichtenberg, F., Cheever, A. W., and Erickson, D. G. (1970). Schistosomiasis mansoni in the chimpanzee. The natural history of chronic infections after single and multiple exposures. American Journal of Tropical Medicine and Hygiene, 19, 258-277.

Schiff, L. (1975). Diseases of the Liver. Lippincott: Philadelphia.

Sherlock, S. (1975). Diseases of the Liver and Biliary system, Davis: Philadelphia.

Sulit, Y. S. M., Domingo, E. O., Dalmacio-Cruz, A. E., De Peralta, D. S., and Imperial, E. S. (1964). Parasitic cirrhosis among Filipinos. Journal of the Philippine Medical Association, 40, 1021-1038.

Symmers, W. St. C. (1904). Note on a new form of liver cirrhosis due to the presence of the ova of Bilharzia haematobia. Journal of Pathology and Bacteriology, 9, 237-239.

Von Lichtenberg, F., Sadun, E. H., Cheever, A. W., Erickson, D. G., Johnson, A. J., and Boyce, H. W. (1971). Experimental infection with Schistosoma japonicum in chimpanzees. Parasitologic, clinical, serologic, and pathological observations. American Journal of Tropical Medicine and Hygiene, 20, 850-893.

Warren, K. S. (1962). The influence of treatment on the development and course of murine hepato-splenic schistosomiasis mansoni. Transactions of the Royal Society of Tropical Medicine and Hygiene, 56, 510-519.

Warren, K. S. (1966). The pathogenesis of clay pipe-stem cirrhosis' in mice with chronic schistosomiasis mansoni, with a note on the longevity of the schistosomes. American Journal of Pathology, 49, 477-489.

Warren, K. S. (1972). The immunoparhogenesis of schistosomiasis: A multidisciplinary approach. Transactions of the Royal Society of Tropical Medicine and Hygiene, 66, 417-434.

Warren, K. S. (1973a). The pathology of schistosome infections. Helminthological Abstracts, 42, 591-633.

Warren, K. S. (1973b). Regulation of the prevalence and intensity of schistosomiasis in man: Immunology or ecology? Journal of Infectious Diseases, 127, 595-609.

Warren, K. S. (1975). Hepatosplenic schistosomiasis mansoni: An immunologic disease. Bulletin of the New York Academy of Medicine, 51, 545-550.

Warren, K. S. (1977). Modulation of immunopathology and disease in schistosomiasis. American Journal of Tropical 
Medicine and Hygiene, 26, Suppl. (Nov)., 113-119.

Warren, K. S. (1978). The pathology, pathobiology and pathogenesis of schistosomiasis. Nature. (In press.)

Warren, K. S., and DeWitt, W. B. (1958). Production of portal hypertension and esophageal varices in the mouse. Proceedings of the Society for Experimental Biology and Medicine, 98, 99-101.

Warren, K. S., Domingo, E. O., and Cowan, R. B. T. (1967). Granuloma formation around schistosome eggs as a manifestation of delayed hypersensitivity. American Journal of Pathology, 51, 735-756.

Warren, K. S., and Hoffman, D. B., Jr. (1976). Schistosomiasis III. Abstracts of the Complete Literature 1963-1974. Hemisphere Publishing Corporation: Washington, D.C., and London.

Warren, K. S., and Klein, L. (1969). Chronic murine hepatosplenic schistosomiasis mansoni: relative irreversibility after treatment. Transactions of the Royal Society of Tropical Medicine and Hygiene, 63, 333-337.
Warren, K. S., and Moore, D. E. (1966). Murine hepatosplenic schistosomiasis japonica. American Journal of Tropical Medicine and Hygiene, 15, 22-27.

Warren, K. S., and Newill, V. A. (1967). Schistosomiasis: A Bibliography of the World's Literature from 1852-1962. (2 Vols.) The Press of Western Reserve University: Cleveland, Ohio.

Warren, K. S., and Rebouças, G. (1964). Blood ammonia during bleeding from esophageal varices in patients with hepatosplenic schistosomiasis. New England Journal of Medicine, 271, 921-926.

Warren, K. S., Rebouças, G., and Baptista, A. G. (1965). Ammonia metabolism and hepatic coma in hepatosplenic schistosomiasis. Patients studied before and after portavacal shunt. Annals of Internal Medicine, 62, 1113-1133.

Warren, K. S., Rosenthal, M. S., and Domingo, E. O. (1969). Mouse hepatitis virus $\left(\mathrm{MHV}_{\mathbf{3}}\right)$ infection in chronic murine schistosomiasis mansoni. Bulletin of the New York Academy of Medicine, 45, 211-224. 